Research Paper

\title{
Co-delivery of Cyclopamine and Doxorubicin Mediated by Bovine Serum Albumin Nanoparticles Reverses Doxorubicin Resistance in Breast Cancer by Down-regulating P-glycoprotein Expression
}

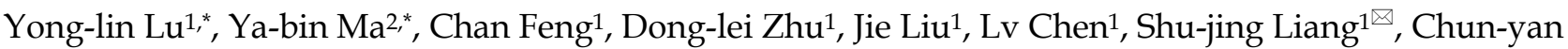 \\ Dong $^{1 凶}$ \\ 1. Breast Cancer Center, Shanghai East Hospital, Tongji University, Shanghai 200120, PR China \\ 2. Department of Pharmacy, Shanghai East Hospital, Tongji University, Shanghai 200120, PR China \\ *Authors contributed equally to this work. \\ $\triangle$ Corresponding author: Chun-yan Dong: Telephone Number: +86-13370029736; Fax number: 021-61569616; E-mail: cy_dong@tongji.edu.cn or Shu-jing Liang: \\ Telephone Number: +86-18817821990; Fax number: 021-61569538; E-mail: liangshujing02@126.com \\ (c) Ivyspring International Publisher. This is an open access article distributed under the terms of the Creative Commons Attribution (CC BY-NC) license \\ (https://creativecommons.org/licenses/by-nc/4.0/). See http://ivyspring.com/terms for full terms and conditions.
}

Received: 2018.10.01; Accepted: 2019.04.12; Published: 2019.05.26

\begin{abstract}
Combination chemotherapy is considered to be one of the most effective treatments for breast cancer by reducing the emergence of drug resistance. In this study, a novel drug delivery system based on bovine serum albumin nanoparticles (BSA NPs) was successfully developed. Doxorubicin (DOX) and cyclopamine (CYC), a potential anti-cancer agent that inhibits the hedgehog signaling pathway were entrapped into BSA NPs through electrostatic interactions and hydrophobic interactions, respectively. Rather than simple combination of two different chemotherapeutics, the CYC also increased the intracellular DOX accumulation by decreasing the expression of P-glycoprotein (P-gP), which could thus reverse the DOX resistance. Tumor-targeting property of nanoparticles was the prerequisite for its further application. Interestingly, retention of fluorescently-labeled particles in vivo indicated that the dual-drug-loaded BSA NPs could not only target the primary tumors, but also target the metastatic lymph nodes, which would simultaneously inhibit the tumor growth and distant metastasis. Taken together, this study provides a promising strategy for co-delivery of drugs, tumor and metastatic lymph node targeting, and DOX resistance reversing in breast cancer chemotherapy.
\end{abstract}

Key words: cyclopamine, doxorubicin, P-glycoprotein, bovine serum albumin nanoparticles, drug resistance

\section{Introduction}

Despite intensive studies were performed on breast cancer, it is still the most common malignant disease with high mortality in women [1]. Doxorubicin (DOX), a conventional chemotherapy drug, is a member of the anthracycline family that has been proven to be effective for breast cancer treatment [2]. But the acquired resistance to DOX has seriously impaired chemotherapeutic effects, which became a major barrier for its further application. A primary mechanism of the development of DOX resistance was the up-regulation of ATP-binding cassette (ABC, a functional protein super-family that pumps foreign substances, including chemotherapeutic drugs, out of cells) [3]. Thus, DOX could be combined with ABC inhibitors to obtain a synergistic effect in breast cancer treatment [4-6].

Cyclopamine (CYC), a steroidal alkaloid that is derived from the veratrum plant, has shown a promising anticancer effect in numerous preclinical studies [7]. It is an inhibitor of the hedgehog $(\mathrm{HH})$ signaling pathway. Aberrant activation of the $\mathrm{HH}$ signaling pathway is responsible for the emergence of multiple human cancers, such as colorectal cancer, lung cancer, and breast cancer [8-10]. In addition, it 
has been reported that the $\mathrm{HH}$ signaling pathway could regulate the expression of $A B C$ sub-family $B$ member 1 (ABCB1, also known as P-glycoprotein (P-gp)) [11, 12]. Indeed, CYC inhibited the $\mathrm{HH}$ signaling pathway by antagonizing the function of the transmembrane protein smoothened (SMO) [13], simultaneously inducing the decrease of P-gp expression (Scheme 1B) [14].

Previous studies have demonstrated that combined application of DOX and $\mathrm{HH}$ signaling pathway inhibitors, reversed DOX resistance by decreasing the expression of P-gp [15]. Nevertheless, the results were not usually satisfactory enough due to different pharmacokinetic properties of different drugs. In this regard, nanotechnology is another alternative method for its unique advantages. Nanoparticles (NPs) carry different drugs efficiently without changing their therapeutic efficacy, and controlled-release drugs release upon reaching the tumor site, thus reducing associated adverse effects [16, 17]. Bovine serum albumin (BSA) has drawn great attention as a nontoxic material with excellent biocompatibility and biodegradability [18]. Comparing to lipids and other organic materials, BSA showed greater drug entrapment and a better ability to prolong drug release time $[19,20]$. The charged amino and carboxylic groups in BSA make it easier to absorb both positively and negatively charged molecules [21], which made it implementable to co-load drugs with different

A

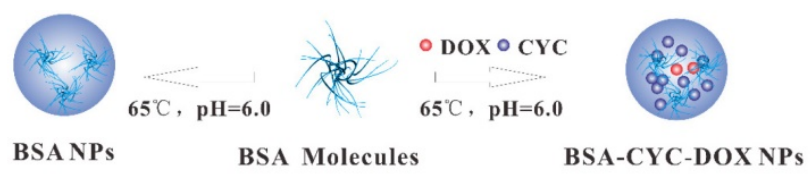

B

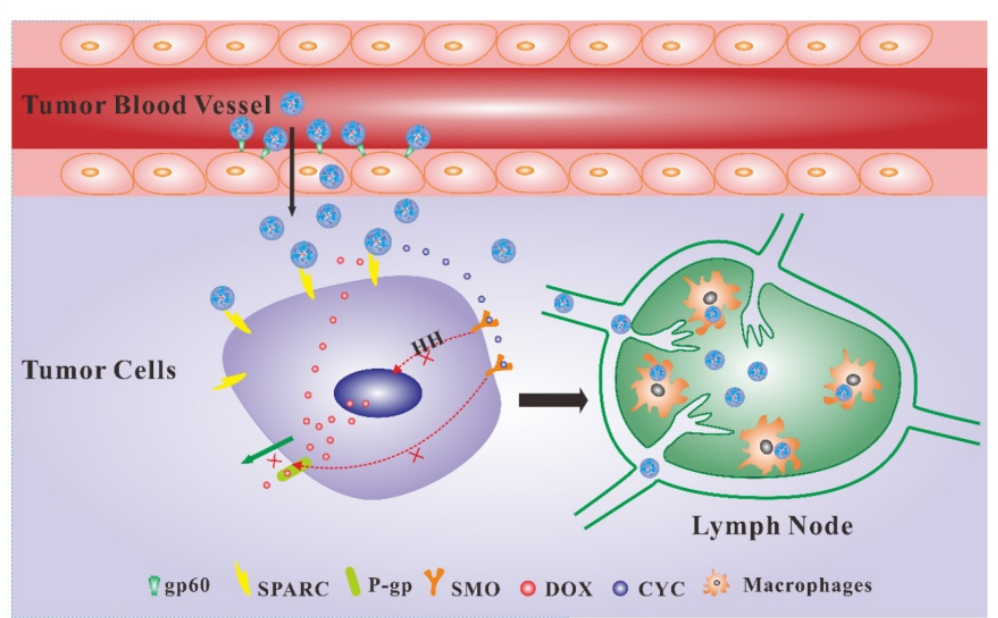

Scheme 1. Schematic illustration of albumin nanoparticles preparation and their targeted delivery to breast cancer cells and metastatic lymph node. (a). The synthesis process of BSA-CYC-DOX NPs and BSA NPs. (b). The dual-drug-loaded NPs were delivered to breast cancer via the active targeting mediated by BSA-binding proteins gp60 and SPARC. CYC increased DOX accumulation by inhibiting $\mathrm{HH}$ signaling pathway and reducing P-gP expression. BSA-CYC-DOX NPs were drained into the metastatic lymph node through lymphatic vessels and retained in macrophages. solubility, such as hydrophobic CYC and hydrophilic DOX. Furthermore, it has been reported that albumin receptors, the glycoprotein 60 (gp60) and secreted protein acidic and rich in cysteine (SPARC) are overexpressed in many tumors including breast cancer [22, 23]. Increased uptake of albumin mediated by gp60 and SPARC would promote the accumulation of BSA NPs in tumor tissues (Scheme 1B).

Herein, we synthesized a novel BSA-based nanoparticles co-loaded with CYC and DOX (BSA-CYC-DOX NPs) for breast cancer treatment. Breast cancer cells (MDA-MB-231), which easily developed resistance to DOX, were used as models. Anti-tumor assays in vitro and in vivo were carried out to prove our hypothesis that, CYC could increase the intracellular DOX accumulation by down-regulating P-gp expression, and that the sufficient amount of DOX and CYC could effectively inhibit tumor growth by eradicating breast cancer cells.

\section{Materials and methods}

\section{Main materials}

Bovine serum albumin (BSA) was purchased from Aladdin Industrial Co. Ltd. (Shanghai, China). Cyclopamine was obtained from Hitsann Biotechnology Co. Ltd. Doxorubicin hydrochloride (DOX $\cdot \mathrm{HCl})$ was supplied by Hvsf United Chemical Materials Co. Ltd. (Beijing, China). Phosphate Buffered Saline (PBS), Dulbecco's Modified eagle's medium (DMEM/high glucose) and trypsin-EDTA were purchased from HyClone (USA). Fetal bovine serum (FBS) and BODIPY 650/665-X NHS Ester (Succinimidyl Ester) were obtained from Lifetechnologies (USA). Cell counting Kit-8 (CCK-8) was purchased from Jiangsu KeyGEN BioTECH Corp. Ltd. (Jiangsu, China). 2-(N-Morpholino) ethanesulfonic acid (MES) was obtained from Sigma-Aldrich. Ethanol and dimethyl sulfoxide (DMSO) were obtained from Sinopharm Chemical Reagent Co. Ltd. Anti-P-glycoprotein and anti-caspase-3 antibody were purchased from Abcam (ab168337, ab13847, UK). BALB/c nude mice were purchased from Silaike Experimental Animal Centre (Shanghai, China). This study was approved by the Ethics Committee of Shanghai East Hospital.

\section{Synthesis of BSA NPs}

Thermal denaturation property of BSA was utilized to prepare BSA NPs. Briefly, $20 \mathrm{mg}$ of BSA was dissolved in 10 $\mathrm{mL}$ MES buffer solution $(50 \mathrm{mM}, \mathrm{pH}=$ 
6.0). The solution was filtered using a $220 \mathrm{~nm}$ nitrocellulose membrane to remove aggregates of albumin. BSA NPs were prepared by heating the solution in $65^{\circ} \mathrm{C}$ water bath under high-speed stirring (750 rpm) within 35 second. Size of the NPs was controllable depending on different heating time.

\section{Synthesis of drugs-loaded BSA NPs}

$5 \mathrm{mg}$ of CYC was dissolved in $1 \mathrm{~mL}$ of ethanol, and $2 \mathrm{mg}$ of DOX was dissolved in $1 \mathrm{~mL}$ of deionized water. $100 \mu \mathrm{L}$ solution of DOX and $100 \mu \mathrm{L}$ solution of CYC were dropwise added into $2 \mathrm{~mL}$ of BSA solution subsequently. BSA-CYC-DOX NPs were prepared as described above: the mixture of BSA ,CYC and DOX were magnetically stirred at $750 \mathrm{rpm}$ in $65^{\circ} \mathrm{C}$ water bath, and the reaction was terminated within 30 second. Ultrafiltration was repeated thrice at 4500 rpm for 30 minutes with deionized water, to wipe out redundant MES and unloaded drugs. BSA-CYC NPs and BSA-DOX NPs were prepared by the same procedure. All the NPs were collected and stored at $4^{\circ} \mathrm{C}$ after being freeze-dried.

\section{Preparation of Bodipy-labeled BSA NPs and BSA-CYC-DOX NPs}

Bodipy 650/665-X NHS Ester was dissolved in DMSO firstly $(10 \mathrm{mg} / \mathrm{mL})$. Then albumin nanoparticles were redissolved in deionized water and blended with Bodipy in a molar ratio of 1:1. Lastly, mixed solution was stirred constantly for 4 hours at room temperature under protection from light. After ultrafiltration and lyophilization (as above), Bodipy-labeled BSA NPs and BSA-CYC-DOX NPs were collected and kept at $4^{\circ} \mathrm{C}$.

\section{Characterization of BSA NPs and BSA-CYC-DOX NPs}

The size distribution and stability of the NPs was measured by the dynamic laser light scattering (DLS, Malvern Instruments, UK) with number size and polydispersity index as evaluation parameters. The morphology was further observed using the transmission electron microscope (TEM, FEI Tecnai G2 F20 S-Twin). Samples for TEM observation were prepared by dipping a carbon-coated copper grid into NPs solution followed by drying in air at room temperature $\left(25^{\circ} \mathrm{C}\right)$.

\section{Determination of drug loading rate}

Minusing was adopted to calculate CYC loading rate. BSA-CYC-DOX NPs was synthesized in MES solution, the synthetic products were filtered and washed thrice with deionized water. Ultrafiltrate containing unloaded CYC was freezed-dried and resuspended in absolute ethanol to remove MES and unassembled BSA. Ethanol was dried in vacuum drier for $24 \mathrm{~h}$ to get the residual CYC. Trace amounts of CYC was analyzed by liquid chromatography mass spectrometry(LC-MS, Waters Acquity TQD, USA) with an acetonitrile water solution containing $0.1 \%$ formic acid as the mobile phase. DOX was tested by UV spectrophotometry (Amersham Pharmacia Biotech, Switzerland).Standard curve of DOX was established by dissolving DOX in PBS and then diluting into different concentration using doubling dilution. Drug loading (DL) rates of CYC and DOX were calculated using the following two formulas, respectively:

$$
\begin{gathered}
\text { DL of CYC }(w / w \%)=\left(W_{0}-W_{\mathrm{t}}\right)_{\mathrm{CYC}} / W_{\mathrm{s}} \times 100 \% \\
\text { DL of DOX }(w / w \%)=W_{\mathrm{DOX}} / W_{\mathrm{s}} \times 100 \%
\end{gathered}
$$

$\mathrm{W}_{0}$ and $\mathrm{W}_{\mathrm{t}}$ are the weight of initial CYC and that of the unencapsulated CYC, respectively. $W_{\mathrm{s}}$ is the weight of BSA-CYC-DOX NPs. $\mathrm{W}_{\text {DOx }}$ is the weight of encapsulated DOX.

\section{In vitro drug release}

Release behavior of dual-drug-loaded NPs was investigated in PBS at $37^{\circ} \mathrm{C}$ under moderate stirring. The concentrations of $\mathrm{CYC}$ and DOX released from BSA-CYC-DOX NPs were measured by LC-MS and UV spectrophotometry. Briefly, $20 \mathrm{mg}$ of BSA-CYC-DOX NPs were suspended in $5 \mathrm{~mL}$ of PBS and transferred into a dialysis tube. Then the dialysis tube was put into a beaker containing $50 \mathrm{~mL}$ of PBS and magnetically stirred at a rate of $200 \mathrm{rpm}$ at $37^{\circ} \mathrm{C} .2$ $\mathrm{mL}$ of PBS containing released drugs was extracted every 2 hours and $2 \mathrm{~mL}$ of fresh PBS was added into the beaker to keep the solution volume. The concentrations of CYC and DOX were analyzed as mentioned above and the percentages of released drugs were calculated based on the cumulative amount of CYC and DOX.

\section{Cell culture}

The human breast cancer lines MDA-MB-231 and MCF-7 were incubated in $25 \mathrm{~mL}$ cell culture flask with high glucose DMEM containing 10\% FBS and 1\% penicillin-streptomycin solution. Cells were cultivated in an incubator at $37^{\circ} \mathrm{C}$ with $5 \%$ carbon dioxide.

\section{Cytotoxicity assessment}

The MDA-MB-231 and MCF-7 cells were seeded in 96-well plates with $5 \times 10^{4}$ cells and $100 \mu \mathrm{L}$ of DMEM in each well. After incubation for $24 \mathrm{~h}$, tumor cells were treated by different concentration of BSA NPs, CYC, DOX, CYC + DOX, BSA-CYC NPs, BSA-DOX NPs, BSA-CYC-DOX NPs for extra $48 \mathrm{~h}$. $100 \mu \mathrm{L}$ of DMEM (without FBS) containing $10 \%$ CCK8 was added into each well. After incubation for 4 hours at $37^{\circ} \mathrm{C}$, the absorbance at $450 \mathrm{~nm}$ was measured 
using a standard enzyme linked immunosorbent assay format spectrophotometer. Experiments were repeated thrice and the cell viability percentages were calculated based on the average of each experiment.

\section{Cellular uptake of DOX}

Confocal laser scanning microscopy (CLSM, Nikon A1R, Japan) and flow cytometry (BD FACS Aria II, USA) were used to examine the accumulation of DOX and DOX-loaded NPs in breast cancer cells. MDA-MB-231 cells were incubated in $35 \mathrm{~mm}$ diameter confocal dishes with $15 \times 10^{4}$ cells per dish. After incubating for $24 \mathrm{~h}$, the cells were treated with different concentration of DOX, CYC + DOX and BSA-CYC-DOX NPs (concentration of DOX: $1 \mu \mathrm{mol} / \mathrm{L}$ and $2 \mu \mathrm{mol} / \mathrm{L}$ ). The culture medium was removed after incubating for extra $24 \mathrm{~h}$. The cells were washed thrice with PBS and fixed with $4 \%$ paraformaldehyde. DAPI was added to each dish for cell nucleus staining. Cells were rinsed with PBS to remove excess DAPI. The wavelengths of Ex/Em for DOX were $480 \mathrm{~nm}$ and $590 \mathrm{~nm}$.

For the flow cytometry experiment, tumor cells were incubated in 6-well plates with $20 \times 10^{4}$ cells per well for $24 \mathrm{~h}$. Corresponding concentration of DOX, CYC +DOX, BSA-CYC-DOX NPs (concentration of DOX: $1 \mu \mathrm{mol} / \mathrm{L}$ and $2 \mu \mathrm{mol} / \mathrm{L}$ ) were added into each well. After incubating for extra $24 \mathrm{~h}$, the cells were harvested and washed thrice with PBS. Autofluorescence intensity of DOX accumulated in cells was measured using flow cytometry.

\section{Breast cancer xenograft model in nude mice}

To establish the tumor xenograft model, $5 \times 10^{5}$ MDA-MB-231 cells were injected into the right mammary fat pad of the mice firstly. When the tumors volume reached $200 \mathrm{~mm}^{3}$, tumors were isolated and divided into small pieces (approximately $1 \mathrm{~mm}^{3}$ ). The breast fat pad orthotopic transplantation of the tumor pieces were conducted using a puncture needle to get a similar initial tumor volume [24].

\section{In vivo anti-tumor activity}

Tumor xenograft model was established on BALB/c female nude mice (4-5 weeks old) by breast fat pad orthotopic transplantation of MDA-MB-231 tissue block. The tumor-bearing mice were randomly divided into 5 groups (4 in each): PBS, CYC, DOX, CYC + DOX, BSA-CYC-DOX NPs, when the tumor volume reached approximately $100 \mathrm{~mm}^{3}$. Intravenous injection of chemotherapy drugs was conducted at a 2-day interval (CYC: $20 \mathrm{mg} / \mathrm{Kg}$, DOX: 2.5 $\mathrm{mg} / \mathrm{Kg}$ ).Tumor volume and mice body weight were monitored every 2 days along with therapy administration. All mice were sacrificed on day 14, and the final tumor weight was measured directly.
Tumor volume was calculated using the following formula:

Tumor volume $=($ length $\times$ width $\times$ width $) / 2$

The tumors and main organs (heart, liver, spleen, lung and kidney) were excised and fixed in $4 \%$ paraformaldehyde for histological examination: hematoxylin and eosin (H\&E) staining and immunohistochemical (IHC) staining.

\section{In vivo distribution of BSANPs and BSA-CYC-DOX NPs}

NightOWL LB 983 IN VIVO imaging system was employed to examine the whole-body fluorescent imaging of tumor-bearing mice after intravenous injection of Bodipy-labeled NPs (Bodipy dose $1 \mathrm{mg} / \mathrm{kg}$ ). Mice injected with PBS, BSA NPs or BSA -CYC-DOX NPs were observed at 1 h, 6 h, 24 h, 36 h and $48 \mathrm{~h}$. Finally, all mice were sacrificed to excise the tumors (or metastatic lymph nodes) and main organs (heart, lung, liver, spleen and kidney) for further observation in vitro.

\section{P-gp expression detected by IHC staining and western blot}

IHC staining of the tumor was performed to analyze expression of P-gp in tumor tissues. The paraffin-embedded tumor sections were incubated overnight at $4^{\circ} \mathrm{C}$ with primary monoclonal antibody against P-gp. Then, the secondary antibody (goat antirabbit 1:100) was added to combine the primary antibody at room temperature. The IHC images were taken by the fluorescence microscope (Nikon ECLIPSE 80i, Japan).

Western blot was conducted to analyze the expression of P-gp in tumor cells in vitro. After treating with certain concentration of different drugs: DMEM containing 10 \% FBS (Control), CYC, DOX, CYC + DOX, BSA-CYC-DOX NPs (concentration of DOX: $1 \mu \mathrm{mol} / \mathrm{L}$, concentration of CYC: $10 \mu \mathrm{mol} / \mathrm{L}$ ) for $24 \mathrm{~h}$, the cells were harvested and lysed with RIPA buffer containing a protease inhibitor cocktail and phosphatase inhibitor cocktail. Protein concentration was determined by the bicinchoninic acid (BCA) method. Protein of different groups were added into the sodium dodecyl sulfate (SDS)-polyacrylamide gels, and transferred to polyvinylidene fluoride (PVDF) membrane. The PVDF membrane was proved with P-gp antibody, followed by HRP-conjugated secondary antibody. The protein bands of different groups were detected by the ECL kit.

\section{Histopathological examination and apoptosis-related protein expression}

Tumors and main organs (heart, liver, spleen, lung and kidney) were excised and fixed in $4 \%$ 
paraformaldehyde for histopathological examination: H\&E staining and IHC staining. After dehydrating in a graded series of alcohol, tumors and organs were embedded in paraffin and tissue sections of each group were stained with hematoxylin-eosin.

Furthermore, apoptosis-related protein caspase3 was detected by IHC staining. The paraffinembedded tumor sections were incubated overnight at $4^{\circ} \mathrm{C}$ with primary anti-caspase- 3 antibody. The secondary antibody (goat antirabbit 1:50) was added to combine the primary antibody at room temperature. The sections of H\&E staining and IHC staining were observed by the optical microscope.

\section{Statistical analysis}

One way single factorial of variance (ANOVA) was performed for all data analysis and values were presented as means \pm standard deviation. $P<0.05\left(^{*}\right)$, $\left.P<0.01{ }^{(*}\right)$ and $P<0.001\left(^{* * *}\right)$ mean significant difference. All the data was analyzed by SPSS 19.0.

\section{Results and Discussion}

\section{Preparation and Characterization of BSA NPs and BSA-CYC-DOX NPs}

A variety of techniques were reported for the synthesis of BSA NPs, such as desolvation and
$\mathbf{A}$

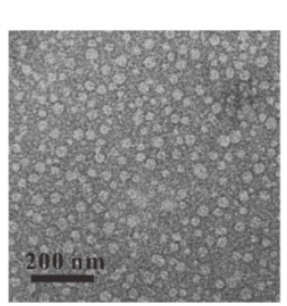

D

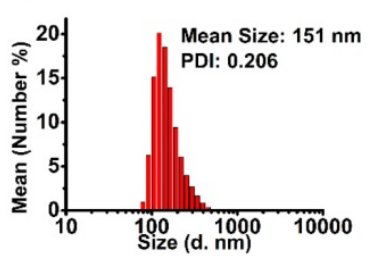

G

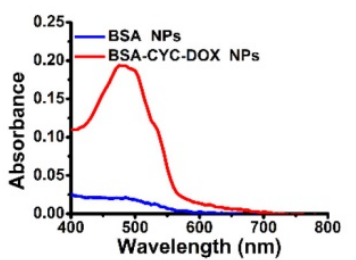

B

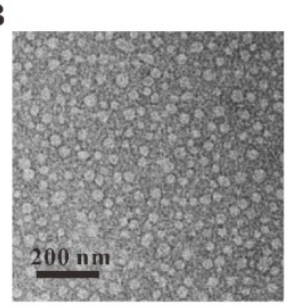

E

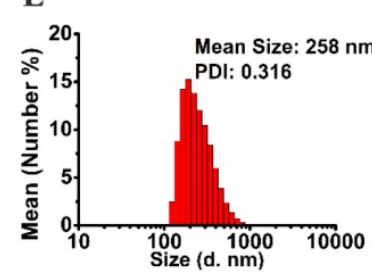

H

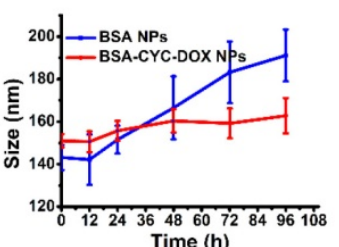

C

F

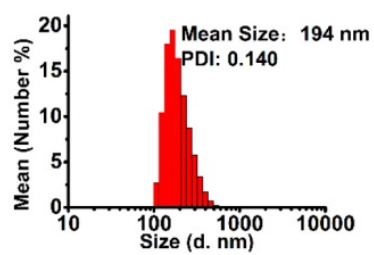

I

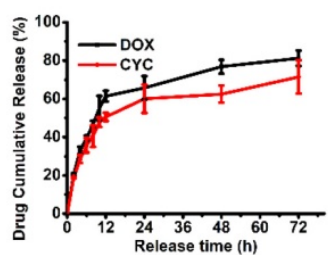

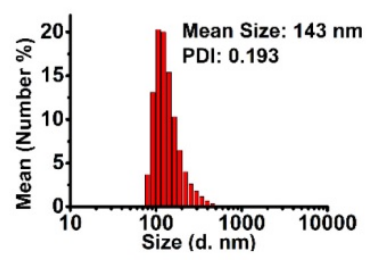

Figure 1. Characterization of BSA NPs and BSA-CYC-DOX NPs. (a-d). Morphology and size distribution measured by TEM and DLS (a, c: BSA NPs. b, d: BSA-CYC-DOX NPs). (e-f). Size distribution of the BSA NPs (e) and BSA-CYC-DOX NPs (f) redissolved in PBS after lyophilization. (g). UV-visible spectra of BSA NPs and BSA-CYC-DOX NPs. (h). Stability of BSA NPs and BSA-CYC-DOX NPs in PBS containing $10 \%$ FBS. (i). In vitro drug release of BSA-CYC-DOX NPs. emulsification [25, 26]. In most of the methods, organic solvents were indispensable, but they could cause serious cytotoxicity. Herein, the dual-drugloaded BSA NPs were synthesized via a simple physicochemical process based on the thermal gelation property of albumin [24]. This process required the heat-induced aggregation of BSA molecules involving the formation of a disulfide bond and hydrogen bond as well as hydrophobic and electrostatic interactions [27]. DOX and CYC were sequentially loaded on the BSA NPs as shown in Scheme 1A.

The morphology and size distribution of the NPs were measured by the transmission electron microscope (TEM) and the dynamic laser light scattering (DLS), respectively. The TEM images (Figure 1A\&B) revealed that the drug-unloaded NPs and drug-loaded NPs presented a spherical structure with uniform size. As shown in Figure 1C, the mean particle size of the BSA NPs in the aqueous solutions was about $143 \mathrm{~nm}$ and the polydispersity index (PDI) was 0.193, while the mean size of the BSA-CYC-DOX NPs was $151 \mathrm{~nm}$ and the PDI was 0.206 (Figure 1D). Nanoparticles of this size were able to evade the clearance of mononuclear phagocyte system and accumulate in tumor tissues efficiently [28]. The diameter of the NPs was smaller on the TEM image than its hydrodynamic diameters in the aqueous solutions, which could be attributed to the shrinkage property of BSA [29]. The lyophilized powders were dissolved in Phosphate Buffered Saline (PBS) at room temperature. The particle size changes after lyophilization and redissolution were further observed by DLS. As shown in Figure 1E\&F, the hydrodynamic diameters and PDI of the BSA NPs increased apparently, while the hydrodynamic diameters of the drug-loaded BSA NPs presented a slight increase with a stable polydispersity.

For the water-soluble drug, DOX hydrochloride was added into the BSA solution prior to the formation of the BSA NPs. UV spectrophotometry was used to detect whether the DOX had been loaded in the BSA NPs, and typical absorbance peaks of DOX-loaded NPs between 450 $\mathrm{nm}$ and $500 \mathrm{~nm}$ were observed 
(Figure 1G). For the water-insoluble drug, CYC was loaded by the desolvation technique, which has been widely used in hydrophobic drug loading, such as paclitaxel and irinotecan, and some newly developed anti-tumor agents [30-32]. LC-MS was utilized to further ensure the CYC loading into the BSA NPs. The DL percentages of DOX and CYC were $1.2 \pm 0.1 \%$ and $9.1 \pm 0.2 \%$, respectively.

\section{The stability and in vitro release behavior}

The stability of BSA NPs and BSA-CYC-DOX NPs was tested in PBS containing 10\% fetal bovine serum (FBS). As shown in Figure $\mathbf{1 H}$, the size of BSA-CYC-DOX NPs kept stable while the size of BSA NPs slightly increased after $96 \mathrm{~h}$. The improved stability of NPs could be ascribed to the enhanced hydrophobic interaction between the CYC and BSA molecules, which prevented further aggregation of the BSA molecules [33]. The release behavior of BSA-CYC-DOX NPs was investigated in PBS at $37^{\circ} \mathrm{C}$. As shown in Figure 1I, release of DOX and CYC increased rapidly in $12 \mathrm{~h}$, up to $61.4 \%$ and $50.6 \%$ respectively. The release curve indicated that the drugs could reach their effective concentration easily as long as the vehicles arrive at tumor sites quickly.

\section{In vitro anti-tumor efficacy}

The human breast cancer cell line MDA-MB-231 and MCF-7 were used to evaluate the cytotoxicity of
A

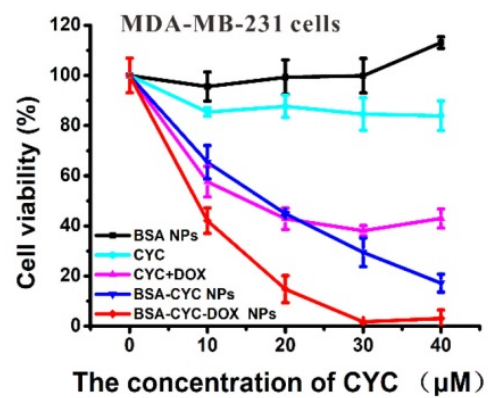

C

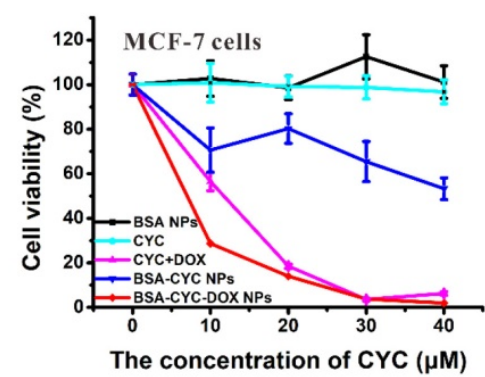

B

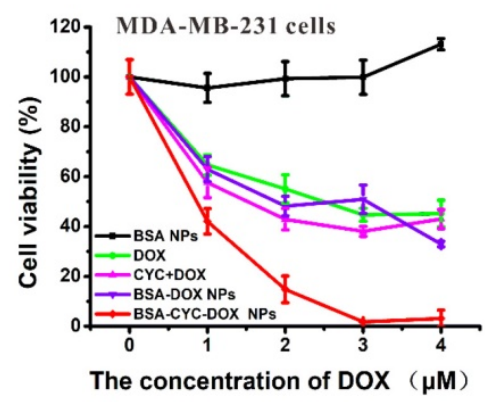

D



Figure 2. Growth inhibition of MDA-MB-231 and MCF-7 cells treated with different drug dosages for $48 \mathrm{~h}$. (a-b). The growth inhibition curve of MDA-MB-231 cells depending on different CYC (a) and DOX (b) dosages. (c-d). The growth inhibition curve of MCF-7 cells depending on different CYC (c) and DOX (d) dosages. the drug-loaded BSA NPs. MDA-MB-231 cells, as the model of triple-negative breast cancer, were reported to easily develop resistance to DOX due to the existence of cancer stem cells and the activation of the $\mathrm{HH}$ signaling pathway [34, 35]. To investigate the cytotoxicity of mono-drug therapy and dual-drug therapy, a cell proliferation assay was divided into two subgroups [36]: subgroup A (BSA NPs, CYC, CYC + DOX, BSA-CYC NPs, and BSA-CYC-DOX NPs) and subgroup B (BSA NPs, DOX, CYC + DOX, BSA-DOX NPs, and BSA-CYC-DOX NPs). As shown in Figure 2, the cell viability curves were observed depending on the CYC concentration and DOX concentration, respectively. As one component of the bovine serum, the drug-free BSA NPs exhibited a growth-promoting effect in MDA-MB-231 cells and MCF-7 cells as expected, which further verified the safety of BSA. Owing to the improvement of solubility, the BSA-CYC NPs manifested potentiated cytotoxicity comparing to the free CYC in MDA-MB-231 cells (Figure 2A). Free DOX and DOX-loaded particles (DOX, CYC+DOX, BSA-DOX NPs, and BSA-CYC-DOX NPs) exhibited a concentration-dependent cytotoxicity in both MDAMB-231 cells and MCF-7 cells (Figure 2B\&D). Furthermore, comparing with the mono-drug-loaded particles (BSA-CYC NPs and BSA-DOX NPs) and the combination therapy of free drugs (CYC + DOX), the BSA-CYC-DOX NPs exhibited an incomparable ability in the growth-inhibition of MDA-MB-231 cells. When the concentration of CYC reached $30 \mu \mathrm{mol} / \mathrm{L}$ (the corresponding DOX concentration was 3 $\mu \mathrm{mol} / \mathrm{L})$, only about $2 \%$ of MDA-MB-231 cells survived (Figure 2A\&B). Different from MDA-MB-231 cells, MCF-7 cells were more sensitive to DOX. Therefore, there was no significant difference of cytotoxicity between DOX-contained groups (DOX and CYC + DOX) and BSA-CYC-DOX NPs group in MCF-7 cells (Figure 2C\&D). These results indicated that the CYC may reverse DOX resistance to obtain a synergistic effect in MDA-MB-231 cells.

\section{Intracellular DOX accumulation results}

Decreased cellular uptake of DOX was regarded as the principal cause of DOX resistance. To measure the intracellular accumulation of free DOX or DOX-loaded particles, the green fluorescence of DOX and the blue fluorescence of nuclei labeled with 4',6-Diamidino-2- 
Phenylindole, Dihydrochloride (DAPI) were observed by confocal laser scanning microscopy [37]. Different from the uptake test in drug-resistant cell lines, the observation of acquired resistance to DOX required enough incubation time for cell proliferation. The MDA-MB-231 cells were treated with DOX, CYC + DOX, and BSA-CYC-DOX NPs for $24 \mathrm{~h}$. Different concentrations of DOX were applied $(1 \mu \mathrm{mol} / \mathrm{L}$ and 2 $\mu \mathrm{mol} / \mathrm{L})$ to assess the influence of DOX dosage. As shown in the Figure $3 A \& C$, similar DOX signals in the tumor cells treated with DOX and CYC + DOX were observed, suggesting that insoluble CYC could hardly increase the DOX intake. For the BSA-CYC-DOX NPs, the inhibition of P-gp expression, which resulted from the improved solubility of CYC, further led to the DOX fluorescence presenting a remarkable increase. To validate the enhanced cellular uptake of DOX further, the fluorescence intensity was tested by flow cytometry. The MDA-MB-231 cells were incubated with DOX and BSA-CYC-DOX NPs for $24 \mathrm{~h}$ in the same way. For the cells pretreated with $1 \mu \mathrm{mol} / \mathrm{L}$ DOX dosage, the mean fluorescence intensities (MFI) of DOX in the cells treated with BSA-CYC-DOX NPs were much higher than the free drug groups (DOX and CYC + DOX) (Figure 3B). When the concentration of DOX reached $2 \mu \mathrm{mol} / \mathrm{L}$ (the corresponding CYC concentration was $20 \mu \mathrm{mol} / \mathrm{L})$, the fluorescence intensity increased more obviously in the tumor cells incubated with BSA-CYC-DOX NPs than that incubated with DOX (Figure 3D), indicating the increased accumulation of DOX due to the increased CYC concentration [15].

\section{In vivo anti-tumor efficacy}

The anti-tumor assay in vivo was performed based on BALB/c female nude mice bearing MDA-MB-231 tumors. Upon the tumor volume reaching $100 \mathrm{~mm}^{3}$, the mice were intravenous administered with PBS, CYC, DOX, CYC + DOX, and BSA-CYC-DOX NPs every 2 days. Mice were killed on day 14 (Figure 4A). Comparing to the PBS group, the CYC exhibited a weak anti-tumor effect, while the DOX and CYC + DOX groups showed definite anti-tumor activity $(P<0.05)$. The BSA-CYC-DOX NPs had the strongest tumor growth inhibition comparing with the PBS group and the free drugs groups (CYC, DOX, CYC + DOX). As shown in Figure 4B, the BSA-CYC-DOX NPs suppressed tumor growth by $100 \%$, which is significantly higher comparing with DOX alone group, suggesting that dual-drug delivery NPs effectively overcame the acquired resistance to DOX. The tumor weight was measured to evaluate the therapeutic effect. The results matched the tumors volume as expected: tumor weights for the BSA-CYC-DOX group and CYC + DOX group on day 14 were $132 \pm 86 \mathrm{mg}$ and $380 \pm 34$ $\mathrm{mg}$ respectively (Figure $4 \mathrm{C}$ ). We monitored the changes of body weight to assess the systemic toxicity of the chemotherapeutic agents. Mice administrated with BSA-CYC-DOX NPs presented no significant weight loss, whereas the combination therapy of free drugs $(C Y C+D O X)$ reduced the weight of the mice by $13 \%$. Mice receiving mono free drug treatment (CYC and DOX) still had a weight growth curve similar to the PBS group (Figure 4D).

\section{Distribution of BSA NPs and BSA-CYC-DOX NPs in vivo}

To achieve a better therapeutic effect, effective tumor accumulation and retention were integral to the NPs. The most common strategy was the enhanced permeability and retention (EPR) effect, which mediated the passive targeted tumor accumulation [38]. Comparing to passive targeting, active targeting showed better potential in various anti-tumor investigations. As shown in Scheme 1B, BSA targeted tumor cells actively mediated by both gp60 (an albumin-receptor in tumor vascular endotheliocyte) and SPARC (a secreted protein over expressed in breast cancer) [39, 40]. Firstly, albumin binds to gp60, which resulted in the transendothelial transport of BSA. Then, binding with SPARC further increased the intratumoral accumulation of BSA [41-43]. In our study, the bio-distribution of BSA NPs was monitored using fluorescence imaging in vivo. Bodipy with near-infrared emission was labeled on the BSA NPs and BSA-CYC-DOX NPs. The whole-body fluorescence imaging in mice was observed at certain time points $(1 \mathrm{~h}, 6 \mathrm{~h}, 24 \mathrm{~h}, 36 \mathrm{~h}$, and $48 \mathrm{~h}$ ) post intravenous injection of the NPs. As shown in Figure 5A, the NPs effectively accumulated in the tumors at $6 \mathrm{~h}$ post injection, and the fluorescence intensity became strongest at $24 \mathrm{~h}$. Observation lasted for $48 \mathrm{~h}$, and no significant difference was found between the BSA NPs and BSA-CYC-DOX NPs. Mice were killed at the end of the whole-body fluorescence imaging to isolate the main organs and tumors. No fluorescence was observed in the hearts and spleens from the BSA NPsand BSA-CYC-DOX NPs-treated mice (Figure 5B). Although fluorescence was observed in the livers, the fluorescence intensity of BSA was much strong in the tumor tissues, and mean fluorescence intensity slightly increased in mice after injection of BSA-CYC-DOX NPs (Figure 5C). Tumors exhibited the most BSA accumulation as expected via the EPR effect and the active targeting mediated by gp60 as well as SPARC. Fluorescence was presented in livers partly due to its specific function in protein 
metabolism. In addition, Figure 5D showed that metastasis of cervical lymph node occurred during the progression of breast cancer, and BSA-CYC-DOX NPs accumulated obviously in tumor and metastatic lymph node. Albumin nanoparticles were often used for lymph node detection as radiolabeled agents [44]. They transferred to the sentinel lymph node from tumor sites though lymphatic channels, and then were gulfed by the lymph node macrophages resulting in a long period retention [45]. Lymph node metastasis plays an important role in the tumor progression. Therefore, accumulation of BSA-CYCDOX NPs in metastatic lymph node may improve the therapeutic effects by preventing distant metastasis of tumor cells.

\section{P-gp expression in tumor cells and tumor tissues}

One common mechanism of drug resistance was the up-regulation of $A B C$ proteins, including P-gp, the breast cancer resistance proteins (BCRP), and the multidrug resistance protein 1 (MRP1) [46]. Therefore, ABC-mediated drug resistance could be reversed by the down-regulation $\mathrm{ABC}$ proteins. To further elucidate the effect of CYC on P-gp expression, the IHC staining of tumor tissues was performed at the end of the anticancer experiments in vivo. As indicated in Figure 6A, tumors from the mice that received treatment of DOX or CYC + DOX presented an increased expression of P-gp. BSA-CYC-DOX NPs could effectively inhibit the tumor growth by down-regulating the P-gp expression. The MDA-MB-231 cells in our study became resistant to DOX easily after incubation with DOX. To further investigate the synergistic effects of CYC and DOX, cells were treated with CYC, DOX, CYC + DOX, and BSA-CYC-DOX NPs for $24 \mathrm{~h}$ respectively. The expression of P-gp was detected by Western blotting (Figure 6B\&C). Free CYC had little effect on the P-gp expression comparing to the normal control group, while cells pretreated with free drugs containing DOX (DOX and CYC + DOX) had a remarkable increase of P-gp expression. Cells incubated with BSA-CYC-DOX NPs kept a low level of protein expression, which was consistent with the results of the IHC staining.
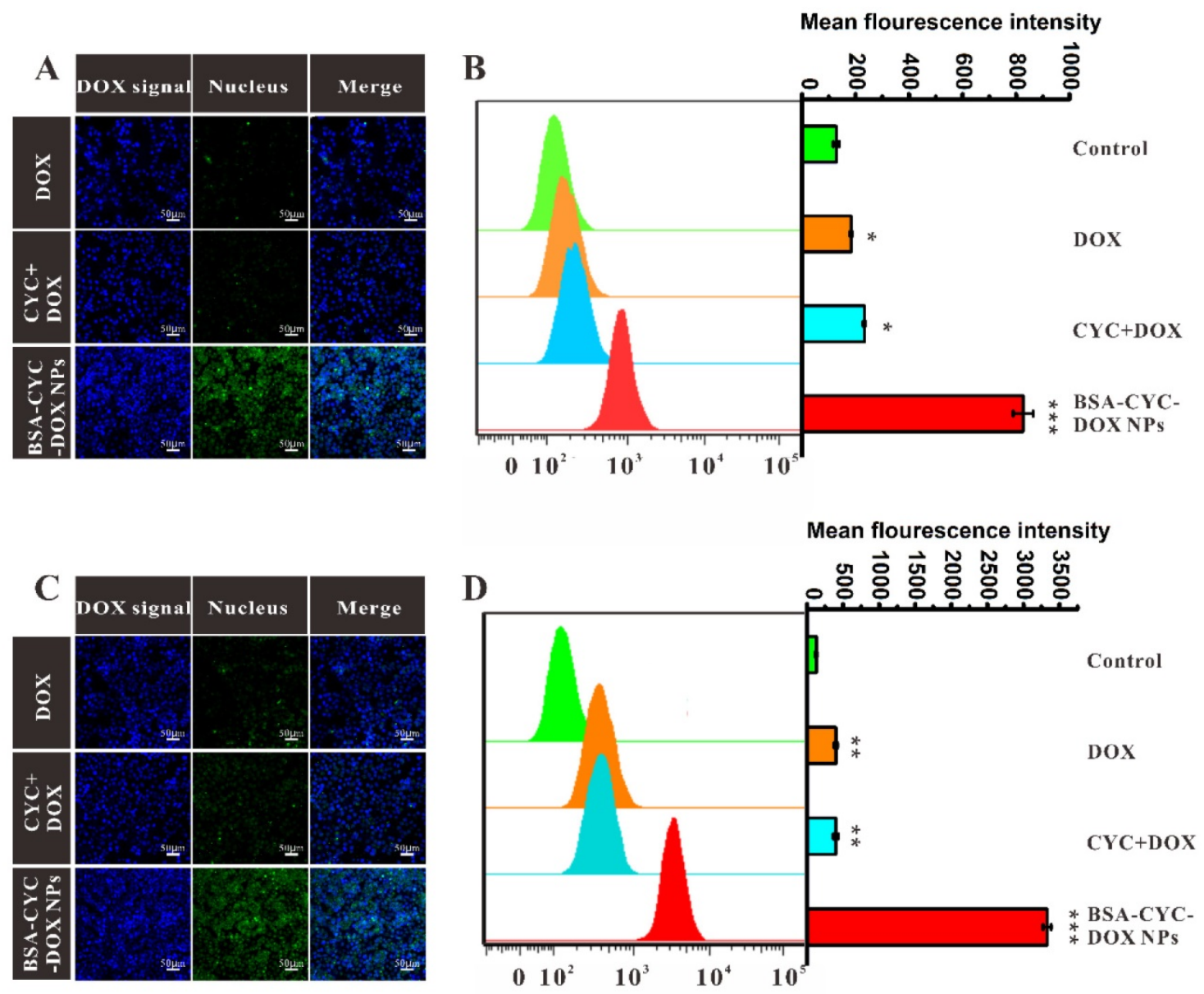

Figure 3. Intracellular accumulation of DOX analyzed by confocal microscope and flow cytometry. (a, c). Confocal microscope images of MDA-MB-231 cells treated with DOX, CYC + DOX, and BSA-CYC-DOX NPs for $24 \mathrm{~h}$ (a: DOX concentration was $1 \mu \mathrm{mol} / \mathrm{L}$. c: DOX concentration was $2 \mu$ mol/L. Scale $=50 \mu \mathrm{m})$. (b, d). The mean fluorescence intensity of MDA-MB-231 cells treated with DOX, CYC + DOX, and BSA-CYC-DOX NPs for 24 h. (b: DOX concentration was $1 \mu$ mol/L. d: DOX concentration was $2 \mu \mathrm{mol} / \mathrm{L})$. 

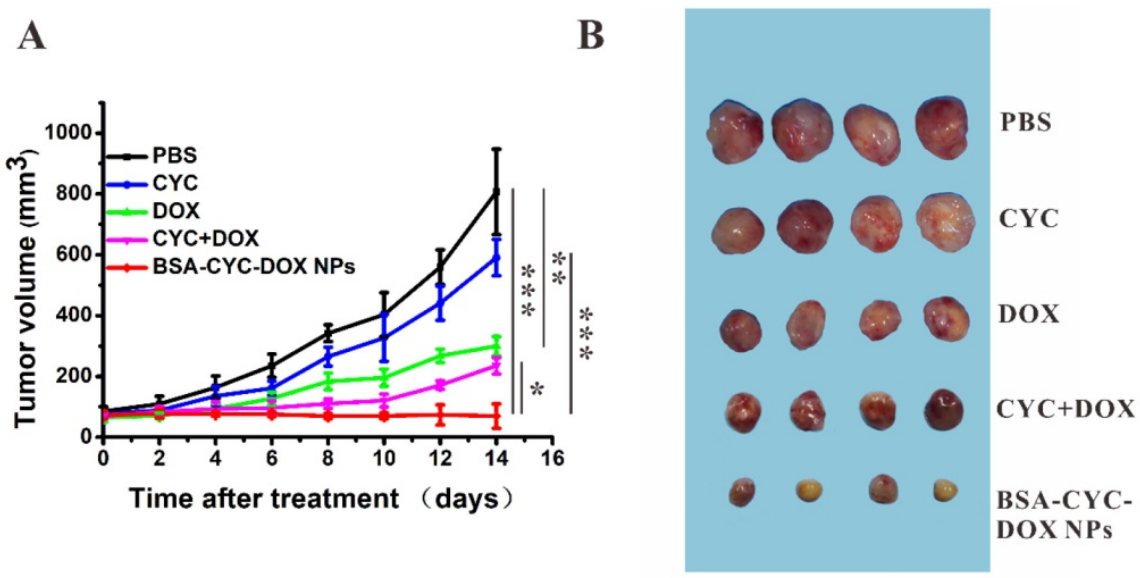

C

D

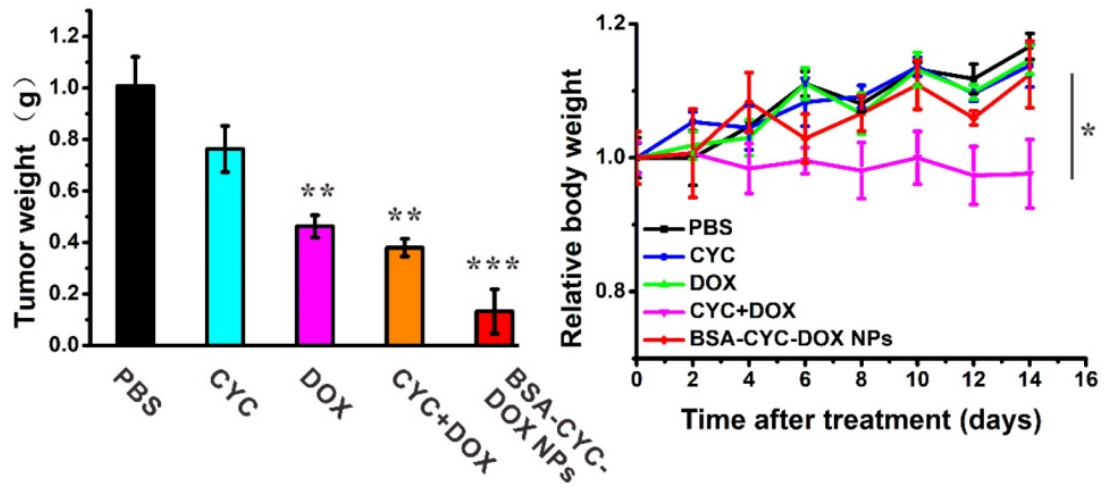

Figure 4. In vivo anti-tumor activity of drugs and drugs-loaded NPs. (a). Tumor growth curves of mice treated with PBS, CYC, DOX, CYC + DOX and BSA-CYC-DOX NPs. (b). The image of tumors isolated from the nude mice on day 14. (c). Histogram of mean tumor weight measured on day 14. (d). The relative body weight of mice. Data were expressed as mean \pm SD $(n=4)$. $* P<0.05$, *** $p<0.01$ and $* * * P<0.001$.

\section{Histopathological examination and caspase-3 expression in tumor tissues}

H\&E staining and IHC staining of the tumors were carried out to analyze that how the combination of CYC and DOX affects the tumor growth. Increased expression of caspase-3, one of the apoptosis-related proteins, was observed in DOX-contained groups (DOX, CYC + DOX) and BSA-CYC-DOX NPs group (Figure 7A). The results indicated that, comparing with the mono therapy of DOX, the dual-drug therapy (CYC + DOX, BSA-CYC-DOX NPs) could inhibit the tumor growth by inducing apoptosis of breast cancer cells.

Histopathological examination of main organs, including heart, liver, spleen, lung and kidney, were conducted to evaluate the toxicity and potential side effects of BSA-CYC-DOX NPs. No apparent histological change was observed in treatment and control groups (Figure 7B). BSA NPs has been proved to be nontoxic by measuring the hepatic and renal function of rats in our previous study [24].

\section{Conclusion}

In summary, an effective anti-breast cancer system, BSA-CYC-DOX NPs, was developed successfully. Co-delivery of CYC and DOX was realized through a self-assembly process of BSA under heating condition. CYC developed a synergistic effect with DOX by reversing its resistance in MDA-MB-231 cell line. Increased intracellular accumulation of DOX resulting from down-regulation of P-gp expression was investigated deeply. Experimental results demonstrated that BSA-CYC-DOX NPs exhibited remarkable anti-breast cancer efficacy in vitro. In addition, comparing with DOX monotherapy, the combination therapy distinctly diminished the tumor size in the xenograft model of nude mice. BSA as a nontoxic and biodegradable carrier has exhibited excellent ability in active targeting of breast cancer cells. BSA-CYC-DOX NPs could rapidly enter tumor tissues before the release of CYC and DOX from carriers. The high bio-distribution of BSA-CYC-DOX NPs at the tumor sites and metastatic lymph nodes could further enhance the therapeutic effects obviously by reducing the distant metastasis of tumor cells. With these attributes, the BSA-based combined application of CYC and DOX could provide a powerful strategy for breast cancer treatment. 
A

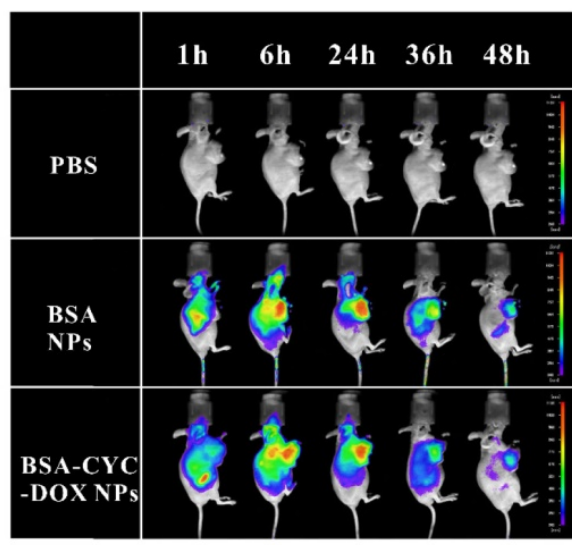

C

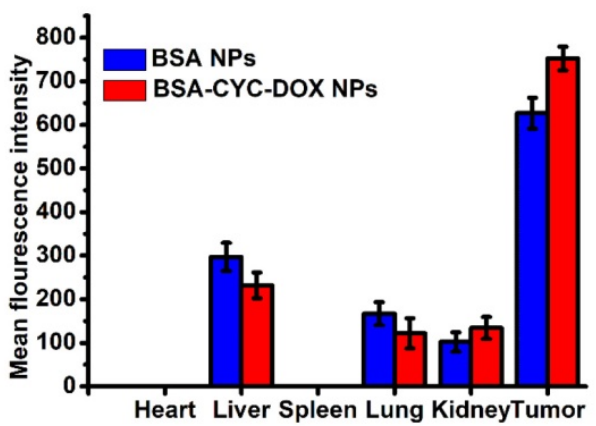

B

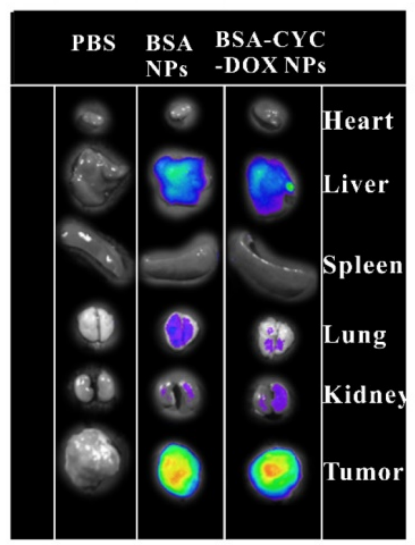

D

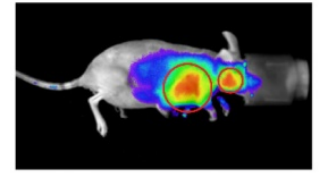

BSA-CYC-DOX NPs

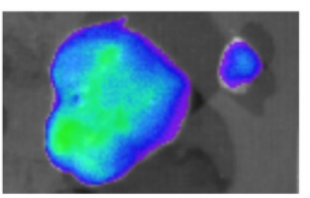

Tumor and metastatic lymph node

Figure 5. The bio-distribution of BSA NPs and BSA-CYC-DOX NPs in tumor-bearing nude mice after intravenous injection of Bodipy-labeled NPs. (a). The whole body fluorescence imaging in mice at $1 \mathrm{~h}, 6 \mathrm{~h}, 24 \mathrm{~h}, 36 \mathrm{~h}$, and $48 \mathrm{~h}$ post injection. (b). The fluorescence imaging of main organs and tumors isolated from mice at 48 $\mathrm{h}$ post injection. (c). The mean fluorescence intensity of main organs and tumors at $48 \mathrm{~h}$ post injection. (d). The fluorescence imaging of tumor and metastatic lymph node after injection of BSA-CYC-DOX NPs.

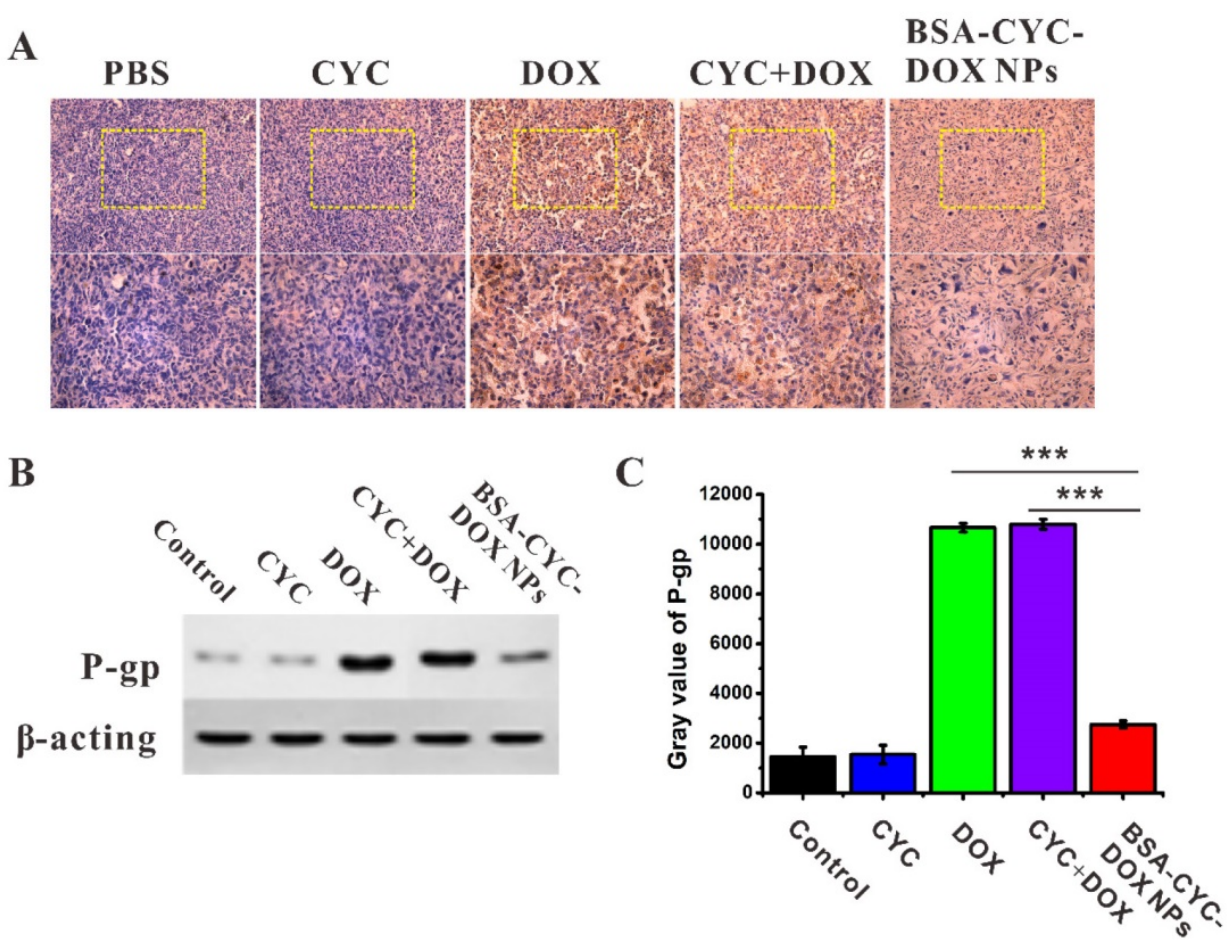

Figure 6. Expression of P-glycoprotein in tumor tissues and tumor cells after various treatments. (a). The expression of $\mathrm{P}$-gP in tumor tissues by IHC staining. (b). The expression of P-gP in tumor cells by western blot assay. (c). Gray value of P-gP bands in western blot assay. $* * * P<0.001$. 
A
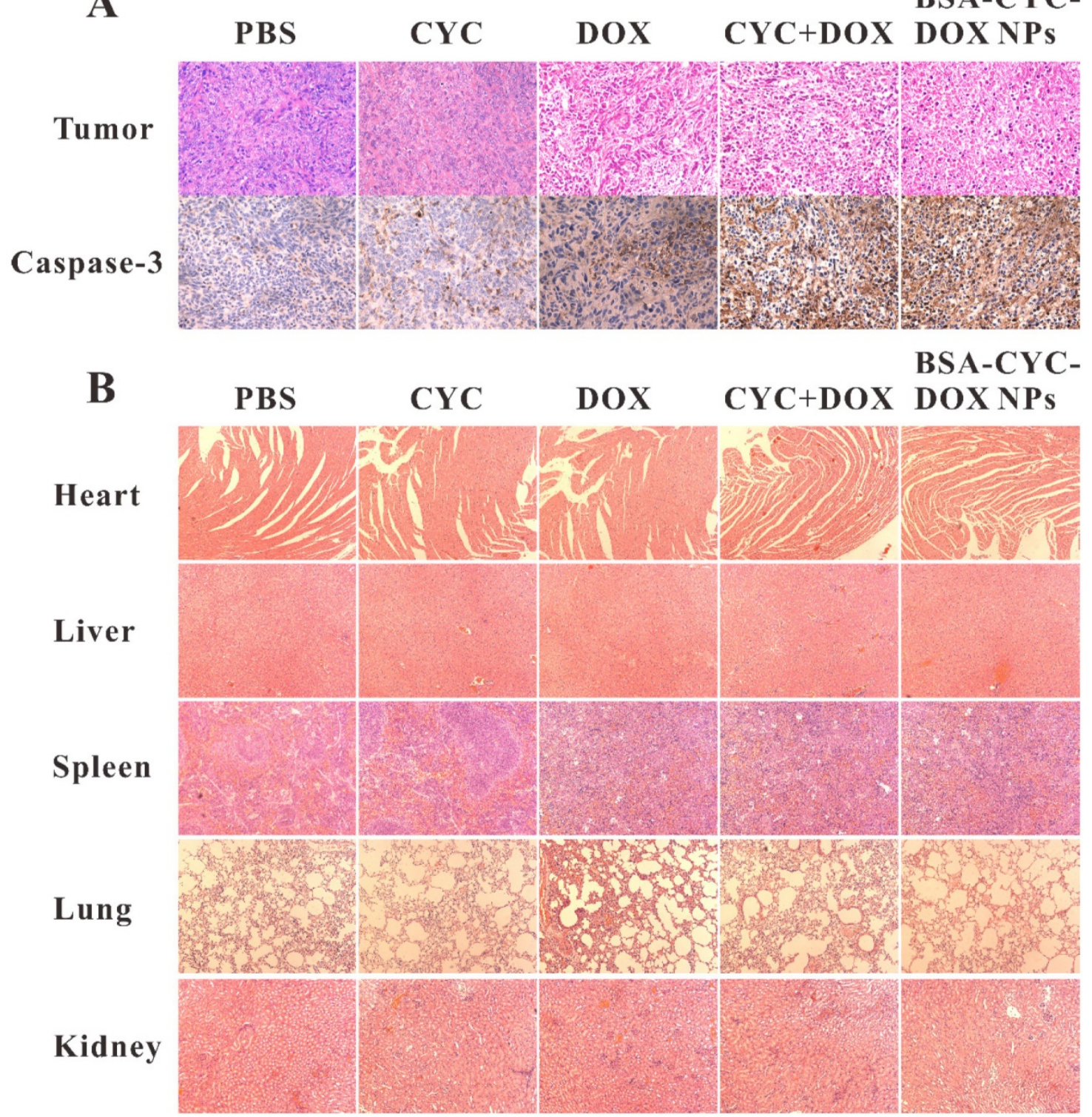

Figure 7. Histopathological examination of tumors and organs isolated from nude mice after different treatments on day 14. (a). H\&E staining of tumor tissues and IHC staining of apoptosis-related protein caspase-3. (b). H\&E staining of main organs.

\section{Abbreviations}

CYC: cyclopamine; DOX: doxorubicin; P-gp: P-glycoprotein; ABC: ATP-binding cassette; $\mathrm{HH}$ : hedgehog; NPs: nanoparticles; BSA: bovine serum albumin; DL: drug loading; gp60: glycoprotein 60; SPARC: secreted protein acidic and rich in cysteine.

\section{Acknowledgements}

This work was supported by the National Nature Science Foundation of China (81860547, 81573008, 81703075), Key Cross Projects of Central Colleges and Universities (1507219075), Project of Excellent Youth Training of Tongji University (2016KJ 064), Key Disciplines Group Construction Project of Pudong Health Bureau of Shanghai (PWZxq2017-13) and Youth Project of Health and Family Planning Committee of Pudong New Area (PW2017B-5).

\section{Competing Interests}

The authors have declared that no competing interest exists.

\section{References}

1. Ferlay J, Soerjomataram I, Dikshit R, Eser S, Mathers C, Rebelo M, et al. Cancer incidence and mortality worldwide: sources, methods and major patterns in GLOBOCAN 2012. Int J Cancer. 2015; 136: 359-86.

2. Yang LJ, Gao Y, Liu JJ, Zhang YM, Ren CH, Wang J, et al. Silver-Coated Nanoparticles Combined with Doxorubicin for Enhanced Anticancer Therapy. J Biomed Nanotechnol. 2018; 14: 312-20.

3. Dvorak P, Hlavac V, Mohelnikova-Duchonova B, Liska V, Pesta M, Soucek P. Downregulation of ABC Transporters in Non-neoplastic Tissues Confers Better Prognosis for Pancreatic and Colorectal Cancer Patients. J Cancer. 2017; 8: 1959-71. 
4. Gao MH, Xu YZ, Qiu LY. Sensitization of multidrug-resistant malignant cells by liposomes co-encapsulating doxorubicin and chloroquine through autophagic inhibition. J Liposome Res. 2017; 27: 151-60.

5. Yang C, Wong ILK, Peng K, Liu Z, Wang P, Jiang TF, et al. Extending the structure-activity relationship study of marine natural ningalin $B$ analogues as P-glycoprotein inhibitors. Eur J Med Chem. 2017; 125: 795-806.

6. Qin Y, Zhang ZM, Huang CL, Fan F, Liu LX, Lu L, et al. Folate-Targeted Redox-Responsive Polymersomes Loaded with Chemotherapeutic Drugs and Tariquidar to Overcome Drug Resistance. J Biomed Nanotechnol. 2018; 14: 1705-18.

7. Drenkhahn SK, Jackson GA, Slusarz A, Starkey NJE, Lubahn DB. Inhibition of Hedgehog/Gli Signaling by Botanicals: A Review of Compounds with Potential Hedgehog Pathway Inhibitory Activities. Curr Cancer Drug Targets. 2013; 13: 580-95.

8. Qualtrough D, Rees P, Speight B, Williams AC, Paraskeva C. The Hedgehog Inhibitor Cyclopamine Reduces beta-Catenin-Tcf Transcriptional Activity, Induces E-Cadherin Expression, and Reduces Invasion in Colorectal Cancer Cells. Cancers. 2015; 7: 1885-99.

9. Zhu DM, Xue WL, Tao W, Li JC. Effects of cyclopamine on the biological characteristics of human breast cancer MCF-7 cell line and its mechanism. Eur J Gynaecol Oncol. 2015; 36: 469-72.

10. Alam MM, Sohoni S, Kalainayakan SP, Garrossian M, Zhang L. Cyclopamine tartrate, an inhibitor of Hedgehog signaling, strongly interferes with mitochondrial function and suppresses aerobic respiration in lung cancer cells. BMC cancer. 2016; 16: 150

11. Malhi V, Colburn D, Williams SJ, Hop CECA, Dresser MJ, Chandra P, et al. A clinical drug-drug interaction study to evaluate the effect of a proton-pump inhibitor, a combined P-glycoprotein/cytochrome 450 enzyme (CYP)3A4 inhibitor, and a CYP2C9 inhibitor on the pharmacokinetics of vismodegib. Cancer Chemoth Pharm. 2016; 78: 41-9.

12. Zhou $\mathrm{H}$, Ma $\mathrm{H}$, Wei W, Ji D, Song X, Sun J, et al. B4GALT family mediates the multidrug resistance of human leukemia cells by regulating the hedgehog pathway and the expression of p-glycoprotein and multidrug resistance-associated protein 1. Cell Death Dis. 2013; 4.

13. Taipale J, Chen JK, Cooper MK, Wang B, Mann RK, Milenkovic L, et al. Effects of oncogenic mutations in Smoothened and Patched can be reversed by cyclopamine. Nature. 2000; 406: 1005-9.

14. Huang FT, Zhuan-Sun YX, Zhuang YY, Wei SL, Tang J, Chen WB, et al. Inhibition of hedgehog signaling depresses self-renewal of pancreatic cancer stem cells and reverses chemoresistance. Int J Oncol. 2012; 41: 1707-14.

15. Liu M, Zhang W, Tang W, Wang Y, Zhao X, Wang X, et al. Isocyclopamine, a novel synthetic derivative of cyclopamine, reverts doxorubicin resistance in MCF-7/ADR cells by increasing intracellular doxorubicin accumulation and downregulating breast cancer stem-like cells. Tumour Biol. 2016; 37: 1919-31.

16. Hu M, Huang P, Wang Y, Su Y, Zhou L, Zhu X, et al. Synergistic Combination Chemotherapy of Camptothecin and Floxuridine through Self-Assembly of Amphiphilic Drug-Drug Conjugate. Bioconjug Chem. 2015; 26: 2497-506.

17. Huang P, Wang D, Su Y, Huang W, Zhou Y, Cui D, et al. Combination of small molecule prodrug and nanodrug delivery: amphiphilic drug-drug conjugate for cancer therapy. J Am Chem Soc. 2014; 136: 11748-56.

18. Thadakapally R, Aafreen A, Aukunuru J, Habibuddin M, Jogala S. Preparation and Characterization of PEG-albumin-curcumin Nanoparticles Intended to Treat Breast Cancer. Indian J Pharm Sci. 2016; 78: 65-72.

19. Wu L, Chen MY, Mao HJ, Wang NN, Zhang B, Zhao XF, et al. Albumin-based nanoparticles as methylprednisolone carriers for targeted delivery towards the neonatal Fc receptor in glomerular podocytes. Int J Mol Med. 2017; 39: $851-60$.

20. Noorani L, Stenzel M, Liang R, Pourgholami MH, Morris DL. Albumin nanoparticles increase the anticancer efficacy of albendazole in ovarian cancer xenograft model. J Nanobiotechnol. 2015; 13: 25.

21. Elzoghby AO, Samy WM, Elgindy NA. Albumin-based nanoparticles as potential controlled release drug delivery systems. J Control Release. 2012; 157: $168-82$.

22. Lin $T$, Zhao $P$, Jiang $Y$, Tang $Y$, Jin $H$, Pan $Z$, et al. Blood-Brain-Barrier-Penetrating Albumin Nanoparticles for Biomimetic Drug Delivery via Albumin-Binding Protein Pathways for Antiglioma Therapy. Acs Nano. 2016; 10: 9999-10012

23. Ding S, Xiong J, Lei D, Zhu XL, Zhang HJ. Recombinant nanocomposites by the clinical drugs of Abraxane((R)) and Herceptin((R)) as sequentially dual-targeting therapeutics for breast cancer. J Cancer. 2018; 9: 502-11.

24. Feng C, Wang K, Lin Y, Song ZW, Lu YL, Liu J, et al. Extracellular retention of a cyclopamine nanoformulation leveraging larger size and more negative charge for improved breast cancer treatment. J Mater Chem B. 2018; 6: 1834-43.

25. Tirkey B, Bhushan B, Uday Kumar S, Gopinath P. Prodrug encapsulated albumin nanoparticles as an alternative approach to manifest anti-proliferative effects of suicide gene therapy. Mater Sci Eng C. 2017; 73: 507-15.

26. Zhao L, Zhou Y, Gao Y, Ma S, Zhang C, Li J, et al. Bovine serum albumin nanoparticles for delivery of tacrolimus to reduce its kidney uptake and functional nephrotoxicity. Int J Pharm. 2015; 483: 180-7.

27. Qi J, Yao P, He F, Yu C, Huang C. Nanoparticles with dextran/chitosan shell and BSA/chitosan core--doxorubicin loading and delivery. Int J Pharm. 2010; 393: 176-84.

28. Lopez-Viota M, El-Hammadi MM, Cabeza L, Prados J, Melguizo C, Ruiz Martinez MA, et al. Development and Characterization of
Magnetite/Poly(butylcyanoacrylate) Nanoparticles for Magnetic Targeted Delivery of Cancer Drugs. AAPS PharmSciTech. 2017; 18: 3042-52.

29. Tsai DH, DelRio FW, Pettibone JM, Lin PA, Tan J, Zachariah MR, et al. Temperature-programmed electrospray-differential mobility analysis for characterization of ligated nanoparticles in complex media. Langmuir. 2013; 29: $11267-74$.

30. Zhao D, Zhao X, Zu Y, Li J, Zhang Y, Jiang R, et al. Preparation, characterization, and in vitro targeted delivery of folate-decorated paclitaxel-loaded bovine serum albumin nanoparticles. Int J Nanomed. 2010; 5: 669-77.

31. You J, Zhao J, Wen X, Wu C, Huang Q, Guan F, et al. Chemoradiation therapy using cyclopamine-loaded liquid-lipid nanoparticles and lutetium-177-labeled core-crosslinked polymeric micelles. J Control Release. 2015; 202: 40-8.

32. Lee EJ, Lim KH. Hardly water-soluble drug-loaded gelatin nanoparticles sustaining a slow release: preparation by novel single-step $\mathrm{O} / \mathrm{W} / \mathrm{O}$ emulsion accompanying solvent diffusion. Bioprocess Biosyst Eng. 2017; 40: 1701-12.

33. Das RP, Singh BG, Kunwar A, Ramani MV, Subbaraju GV, Hassan PA, et al. Tuning the binding, release and cytotoxicity of hydrophobic drug by Bovine Serum Albumin nanoparticles: Influence of particle size. Colloid Surf B-Biointerfaces. 2017; 158: 682-8.

34. Sun M, Yang C, Zheng J, Wang M, Chen M, Le DQS, et al. Enhanced efficacy of chemotherapy for breast cancer stem cells by simultaneous suppression of multidrug resistance and antiapoptotic cellular defense. Acta biomater. 2015; 28: $171-82$.

35. Tan Q, Wang $\mathrm{H}, \mathrm{Hu} \mathrm{Y}, \mathrm{Hu} \mathrm{M}, \mathrm{Li}$ X, Aodengqimuge, et al. Src/STAT3-dependent heme oxygenase-1 induction mediates chemoresistance of breast cancer cells to doxorubicin by promoting autophagy. Cancer Sci. 2015; 106: 1023-32.

36. Hu K, Zhou H, Liu Y, Liu Z, Liu J, Tang J, et al. Hyaluronic acid functional amphipathic and redox-responsive polymer particles for the co-delivery of doxorubicin and cyclopamine to eradicate breast cancer cells and cancer stem cells. Nanoscale. 2015; 7: 8607-18.

37. Hao N, Sun CZ, Wu ZF, Xu L, Gao WX, Cao J, et al. Fabrication of Polymeric Micelles with Aggregation-Induced Emission and Forster Resonance Energy Transfer for Anticancer Drug Delivery. Bioconjugate Chem. 2017; 28: 1944-54.

38. Mei KC, Bai J, Lorrio S, Wang JT, Al-Jamal KT. Investigating the effect of tumor vascularization on magnetic targeting in vivo using retrospective design of experiment. Biomaterials. 2016; 106: 276-85.

39. Ma J, Gao S, Xie X, Sun E, Zhang M, Zhou Q, et al. SPARC inhibits breast cancer bone metastasis and may be a clinical therapeutic target. Oncol Lett. 2017; 14: 5876-82.

40. Zhu A, Yuan P, Du F, Hong R, Ding X, Shi X, et al. SPARC overexpression in primary tumors correlates with disease recurrence and overall survival in patients with triple negative breast cancer. Oncotarget. 2016; 7: 76628-34.

41. He YJ, Xing L, Cui PF, Zhang JL, Zhu Y, Qiao JB, et al. Transferrin-inspired vehicles based on $\mathrm{pH}$-responsive coordination bond to combat multidrug-resistant breast cancer. Biomaterials. 2017; 113: 266-78.

42. Yardley DA. nab-Paclitaxel mechanisms of action and delivery. J Control Release. 2013; 170: 365-72.

43. Yin Q, Tang L, Cai K, Yang X, Yin L, Zhang Y, et al. Albumin as a "Trojan Horse" for polymeric nanoconjugate transendothelial transport across tumor vasculatures for improved cancer targeting. Biomater Sci. 2018; 6: 1189-200.

44. Persico MG, Lodola L, Buroni FE, Morandotti M, Pallavicini P, Aprile C. (99m)Tc-human serum albumin nanocolloids: particle sizing and radioactivity distribution. J Labelled Comp Radiopharm. 2015; 58: 376-82.

45. Subramanian S, Pandey U, Shah S, Rangarajan V, Samuel G. An indigenous single-vial kit formulation of human serum albumin nanocolloid for use in sentinel lymph node detection. Nucl Med Commun. 2015; 36: 848-53.

46. Hermawan A, Wagner E, Roidl A. Consecutive salinomycin treatment reduces doxorubicin resistance of breast tumor cells by diminishing drug efflux pump expression and activity. Oncol Rep. 2016; 35: 1732-40. 